\title{
PROJETO CINOTERAPIA: \\ FORMA DE INTERVENÇÃ̃ NAS NECESSIDADES DE SAÚDE ESPECIAIS
}

\author{
Cátia Rodrigues Mariano \\ Psicóloga Clínica, CASPAE \\ catia.rodrigues@caspae.pt \\ Maria Emília Bigotte de Almeida \\ Presidente de direção, CASPAE \\ caspae@caspae.pt
}

Recepción Artículo: 3 febrero 2020 Admisión Evaluación: 4 marzo 2020

Informe Evaluador 1: 10 m 2020

Informe Evaluador 2: 15 marzo 2020

Aprobación Publicación: 20 abril 2020

\section{RESUMO}

A Perturbação do Espectro do Autismo (PEA) é uma perturbação neurodesenvolvimental caraterizada por défices persistentes na comunicação social e na interação social e por padrões restritos de comportamentos, interesses e atividades. A cinoterapia pode ser definida como uma terapia assistida pelo cão, em que o mesmo exerce a função de facilitador do processo terapêutico. 0 principal objetivo deste projeto é verificar a eficácia da cinoterapia na melhoria dos comportamentos de autorregulação, comunicação e socialização de crianças com PEA, em contexto escolar. Participam 16 crianças com diagnóstico de PEA, que foram avaliadas numa fase pré e pós intervenção. No grupo de controlo o método educativo da Unidades de Ensino Estruturado para a Educação de Alunos com Perturbações do Espetro do Autismo é o modelo Treatment and Educacion of Autistic and Related Communications Handicapped Children (TEACH) e no grupo experimental o método educativo é o mesmo, contudo com 0 apoio do cão ao longo das 40 sessões que têm a duração de 30 minutos. No grupo experimental espera-se que existam melhorias significativas nas medidas de irritabilidade e hiperatividade, nos processos de socialização, cognição social e na comunicação social.

Palavras-chave: perturbação do espectro do autismo; cinoterapia; comunicação; socialização; autorregulação

\section{ABSTRACT}

Kynotherapy project: form of intervention in the needs of special health. Autism Spectrum Disorder (ASD) is a neurodevelopmental disorder characterized by persistent deficits in social communication and interaction as well as by restricted patterns of behavior, interests, and activities. In kynotherapy, a dog it acts as a facilitator of the therapeutic process. This pilot-project has as main objective to study the effectiveness of the 


\section{PROJETO CINOTERAPIA: FORMA DE INTERVENÇ̃̃O NAS NECESSIDADES DE SAÚDE ESPECIAIS}

kynotherapy in the improvement of the behaviors of self-regulation, communication and socialization of children with ASD, in a school context. Of the pilot project, 16 children with a diagnosis of ASD, were evaluated in a preand post-intervention phase. In the control group the educational method of the Structured Teaching is the Treatment and Educacion of Autistic and Related Communications Handicapped Children (TEACH) model and in the experimental group the educational method is the same, however with there is the support of a dog during the 40 sessions, that have the duration of 30 minutes. In the experimental group, it is expected significant improvements in the measures of irritability and hyperactivity, as well as in the processes of socialization, social cognition and social communication.

Keywords: autism spectrum disorder; kynotherapy; communication; socialization; self-regulation

\section{INTRODUÇÃO}

Em Portugal, todos os anos o número de crianças com Necessidades de Saúde Especiais (NSE) aumenta. As perturbações neurodesenvolvimentais são incapacitantes e dificultam a aprendizagem das crianças (Thayer et al., 1998). Cabe a nós profissionais minimizar a disfuncionalidade destas crianças atribuindo-lhes e concedendoIhes competências para promover uma melhor qualidade de vida (Capucha, 2008).

Em 1943, é publicado o primeiro artigo que descreve casos clínicos de crianças com as características da PEA. (Kanner, 1943). A PEA é diagnosticada com base na observação comportamental da criança e nos indicadores de desenvolvimento atípico. Contudo, existem cada vez mais estudos que indicam a base genética e biológica da etiologia do autismo (Yenkoyan, Grigoryan, Fereshetyan, \& Yepremyan, 2017).A PEA é uma perturbação neurodesenvolvimental diagnosticada com base nos défices persistentes na interação social, nos comportamentos comunicativos e por apresentar padrões restritos e repetitivos de comportamentos (APA, 2015). Nestas crianças a comorbilidade com outras perturbações também é frequente, tais como:perturbação de hiperatividade/défice de atenção, perturbações de humor e incapacidade intelectual. Na adolescência, as dificuldades de aprendizagem, a desmotivação, 0 comportamento desafiador e, por vezes, 0 abandono escolar são as características mais frequentes. As crianças com PEA exibem mais comportamentos desafiadores e inapropriados (Matson, Gonzalez, \& Rivet, 2008; Matson, Wilkins, \& Macken, 2008) e apresentam mais sintomas de stress, ansiedade e depressão do que outras crianças com outro género de psicopatologia (Leyfer et al., 2006; White, Oswald, Ollendick, \& Scahill, 2009). Também podem apresentar outras características, tais como a irritabilidade, a hiperatividade, a dispraxia, entre outros (Gabriels et al., 2008). Alguns estudos (Lecavalier, Leone, \& Wiltz, 2006; Schieve, Blumberg, Rice, Visser, \& Boyle, 2007) indicam que os cuidadores de crianças com PEA apresentam índices de stress mais elevados quando comparados com cuidadores de crianças com outras patologias.Assim, a criança está integrada numa família, sendo esta a primeira forma de socialização da criança, promovendo o seu desenvolvimento ao nível social, emocional, físico e cognitivo. Contudo, a criança também faz parte da comunidade, sendo que quando a relação da família com a comunidade entra em desequilíbrio, o desenvolvimento da criança pode também ser alterado (Bronfenbrenner, 1994). Esta perspetiva surge do modelo ecológico de Bronfenbrenner (1994) que defende que a criança deve ser vista como um ser biopsicossocial. Assim, apoiar e fornecer estratégias de intervenção efetivas às crianças com PEA é um desafio quer para a família quer para os profissionais educativos. 0 que propomos com este projeto é a aplicação de uma terapia complementar, promovendo uma melhoria na qualidade de vida da criança com PEA.A relação homem-animal está documentada desde os nossos ancestrais. No entanto, apenas a partir de 1960 devido a uma descoberta de Boris Levinson, começou a ser elaborada alguma investigação sobre os benefícios desta relação. Essas pesquisas fomentaram a que 0 National Institute of Health, em 1987, realizasse um dos workshops mais importantes sobre os benefícios para a saúde humana da relação homem-animal. Este workshop foi um marco histórico na promoção e no desenvolvimento das Intervenções Assistidas por Animais (IAA; NIH, 1987). A ideia de usar o cão em contexto clínico foi evidenciada por cientistas da Psicologia. A filha de Sigmund Freud, em 1937, Anna Freud verificou que na relação homem-cão existem processos semelhantes à compreensão emocional. Também nalguns instrumentos a avaliação se verifica que crianças criam mais facilmente vínculos emocionais com animais do que com adultos. 
Contudo, a reabilitação com recurso ao cão apenas se fundamentou e teorizou em 1960 com Boris Levinson. Boris Levinson percebeu que a presença do cão em contexto terapêutico melhorou a comunicação com o seu utente e acelerou o processo de terapia (Fine, 2010). Foi então proposto incorporar animais, como forma complementar, no processo de intervenção de crianças com PEA para a diminuição da sintomatologia e promoção de comportamentos funcionais e adaptativos (Fine, 2010).

As Intervenções Assistidas por Animais (IAA) podem ser divididas em três tipos: atividades assistidas por animais, a terapia assistida com animais e programas de animais de serviço (Muñoz Lasa et al., 2015). As atividades assistidas por animais são caraterizadas pelo seu carácter genérico.Os programas de animais de serviço cães de assistência - são por exemplo os cães guia que apoiam o deficiente visual no seu dia-a-dia. Já a terapia assistida com animais (TAA) é uma intervenção formal, com eficácia comprovada, com objetivos terapêuticos precisos em que 0 animal age como um facilitador da terapia. Esta é aplicada conjuntamente com um programa de reabilitação que varia consoante o diagnóstico, sendo coordenada por uma equipa multidisciplinar, que regista o progresso do paciente e avalia os resultados (Muñoz Lasa et al., 2015).

A terapia assistida pelo cão com objetivos terapêuticos e/ou educacionais designa se por cinoterapia. Nesta terapia o cão é um mediador do processo de intervenção. Atualmente, esta terapia é utilizada nas diversas ciências para estabelecer uma ponte entre utentes e terapeutas e é utilizado como um estímulo dos órgãos sensoriais, do sentido cinestésico e do sistema límbico (Viau et al., 2010). Por consequência, são desencadeados nos utentes sentimentos de cuidado, confiança, empatia e compaixão (Ciari, Chelini, Lacerda, Rocha, \& Otta, 2013; Fine, 2010). A ligação da criança-cão promove a aquisição de competências comunicacionais, competências prósociais, competências emocionais e promove a sensação de relaxamento (Fine, 1999). № caso específico das crianças com PEA, a cinoterapia promove a aquisição de autonomia, a comunicação pró-social e a melhoria da autoestima. 0 principal objetivo é a generalização destes comportamentos e sentimentos aos outros contextos, não sendo necessária a presença do cão.

A cinoterapia tem a sua eficácia comprovada em perturbações mentais e neurológicas (Kamioka et al., 2014). Por exemplo, num estudo de revisão realizado em 2015 verifica-se que crianças com PEA, após doze semanas de intervenção, aumentaram a sua autoestima, melhoraram as suas relações interpessoais, diminuíram o seu sedentarismo e aumentaram a sua autonomia (Maujean, Pepping, \& Kendall, 2015). Também noutro estudo verifica-se que uma criança que interage com animais cria mais facilmente sentimentos de empatia e compaixão pelos outros, tem melhores estratégias de coping ao stress, melhor autocontrolo e autorregulação emocional (Fine, 2010). A cinoterapia é uma forma de complementar as estratégias terapêuticas mais tradicionais promovendo uma melhor colaboração e envolvimento da criança na terapia (Maujean et al., 2015). Em países como Brasil, Austrália, Noruega, Canadá a cinoterapia está inserida no protocolo de intervenção com crianças com problemas neurodesenvolvimentais (Sousa Magalhães, 2014). Com recurso à cinoterapia, nas crianças com PEA há a diminuição de hormonas responsáveis pelo stress (e.g. cortisol) (Viau et al., 2010) e 0 aumento de comportamentos pró-sociais (Marston, 2011). As crianças com PEA necessitam de intervenções que estimulem e promovam comportamentos pró-sociais e autonomia, que reduzam comportamentos disfuncionais e não adaptativos, quer na infância quer quando forem adultos.

\section{AMOSTRA}

A amostra do estudo é constituída por 16 crianças, das quais 10 são do género masculino e 6 do género feminino. As idades variam entre os 6 e os 11 anos. 0 grupo experimental (GE) é composto por 8 crianças, 5 rapazes e 3 raparigas, com uma média de idades de 8,6 anos $(D P=1,30)$. Quanto às habilitações a média de anos de escolaridade é de $2(\mathrm{DP}=0,99)$. Este grupo além do método educativo TEACH também teve intervenção com o Programa de Intervenção de Cinoterapia (PIC). 0 grupo de controlo (GC) é constituído por 8 elementos, 5 rapazes e 3 raparigas, com média de idade de $8,13(\mathrm{DP}=1,25)$ e uma média de 2 anos de escolaridade. Este grupo teve apenas como método educativo o TEACH. Não existem diferenças estatisticamente significativas entre os grupos em relação às variáveis sociodemográficas apresentadas. Contudo, foram observadas diferenças esta- 


\section{PROJETO CINOTERAPIA: FORMA DE INTERVENÇ̃̃O NAS NECESSIDADES DE SAÚDE ESPECIAIS}

tisticamente significativas do género face à idade, sendo que o género feminino apresenta uma média de idades superior (t (14) $=-2,875 ; p>0,05)$.

\section{METODOLOGIA E INSTRUMENTOS UTILIZADOS}

Relativamente aos instrumentos a utilizar foi elaborado um documento de consentimento informado aos encarregados de educação. Foi aplicado um questionário sociodemográfico e elaborada uma entrevista. A Entrevista foi realizada com os encarregados de educação e professor de ensino especial. Esta entrevista foi orientada pela psicóloga clínica e teve como objetivos conhecer o contexto familiar da criança, identificar quais os comportamentos reforçadores e gratificantes para a criança e quais os comportamentos funcionais e disfuncionais de cada criança. Desta forma, a entrevista assume-se como um instrumento crucial para a avaliação adequada de cada participante de forma a realizar um plano individual de intervenção apropriado (Reis, Pereira, \& Almeida, 2012). Para a avaliação das competências básicas, comunicativas, sociais e linguísticas da criança utilizou-se um guião informal de entrevista (Marinho, Gomes, Vieira, Antunes, \& Teixeira, 2009). Para avaliar o perfil comportamental da amostra utilizou-se a Escala de Avaliação dos Traços Autistícos (ETA; Escala de'avaluacio dels trests autistes, Ballabriga, Escudé e Llaberia, 1994; versão portuguesa de Assumpção, Kuczynski, Gabriel, \& Rocca, 1999) é um instrumento que consiste numa escala de observação direta da criança, composta por vinte e três subescalas, cada uma dividida em diferentes itens Esta é de fácil preenchimento e pode ser aplicada a crianças a partir dos 2 anos de idade. A Escala de avaliação dos traços autistícos (ATA) permite analisar o perfil comportamental da criança longitudinalmente, facilitando 0 registo da evolução e desenvolvimento da criança. No presente estudo 0 alpha Cronbach verificado foi de 0,83, 0 que indica que 0 instrumento tem uma consistência interna boa. Aplicamos também a Escala Revista de Conners para Pais - versão revista (forma reduzida) (SCP; Keith Conners, 1997; versão portuguesa de Rodrigues, 2005) que é um instrumento que avalia os problemas comportamentais de crianças com idades entre os 6 e os 11 anos, tendo a versão para pais e para professores. Neste estudo utilizamos a versão para pais. No presente estudo 0 alpha Cronbach verificado foi 0,71, 0 que indica que 0 instrumento tem uma consistência interna razoável.

0 Programa de Intervenção de Cinoterapia (PIC) tem como base a utilização de diversos jogos lúdicos, que visam estimular a comunicação, as diferentes funções cognitivas, a interação social e uma autorregulação adaptativa. São realizados exercícios, adaptados à capacidade de cada criança, com o objetivo de promover e estimular as competências comunicacionais, promover comportamentos de autorregulação adaptativos e promover 0 aumento comportamentos de socialização. À semelhança de outros estudos (Ciari et al., 2013; Eddy, Hart, \& Boltz, 1988; Pawlik-Popielarska, 2010) o PIC teve a duração de um ano letivo (2016/2017) com um total de 40 sessões de cinoterapia, de 30 minutos e uma vez por semana. As sessões iniciaram-se com um cumprimento da Mel (a cadela de serviço) e com exercícios dinâmicos. No decorrer das sessões, esteve sempre em avaliação se o programa e as suas exigências estavam de acordo com as necessidades da amostra. A equipa multidisciplinar do projeto é constituída pelos encarregados de educação, pela psicóloga clínica, pelo professor de ensino especial, pelo professor titular de turma, pela técnica de terapia assistida por animais, por um treinador de cães e pelo cão de intervenções assistida por animais. Para a elaboração de um plano individual de intervenção adequado tivemos em conta o contexto familiar da criança, ouvindo os encarregados de educação. A análise estatística foi elaborada com recurso ao programa Statistical Package for Social Science (SPSS 20.0). Foram elaboradas estatísticas descritivas e estatísticas inferenciais. Estabeleceu-se um nível de significância de 0,05.

\section{RESULTADOS E CONCLUSÕES}

Apesar de ouvirmos falar cada vez mais das características das crianças com PEA e da possível etiologia da perturbação, os estudos experimentais sobre a eficácia de intervenções com este tipo de população ainda são escassos. Assim, o propósito desta investigação foi avaliar a eficácia da cinoterapia na promoção de comportamentos sociais, comunicacionais e de autorregulação adaptativos em crianças com diagnóstico de PEA. 
Propomos, com este estudo, contribuir empiricamente para a investigação nesta área científica, de forma a implementar a cinoterapia como uma terapia efetiva das Unidades de Ensino Estruturado e do plano educativo individual. Espera-se com este estudo contribuir de forma cientííca na verificação da cinoterapia como uma terapia eficaz na promoção de comportamentos sociais, na promoção da comunicação e na promoção de comportamentos de autorregulação adaptativos.

Redefer e Goodman (1989) foram dos primeiros investigadores que realizaram um estudo experimental que comprova a eficácia da cinoterapia com crianças com diagnóstico de PEA. A corroborar os resultados obtidos nesta investigação está o estudo com crianças diagnosticadas com incapacidade intelectual que demonstrou que 0 uso da cinoterapia contribuiu para que a criança adquirisse comportamentos adaptativos e que os professores verificam uma influência positiva da cinoterapia nas crianças (Pawlik-Popielarska, 2010). À semelhança de outros estudos, os resultados indicam que com a cinoterapia ocorre 0 aumento de contacto com 0 outro, quer com a professora quer com os colegas (Esteves \& Stokes, 2008).

Neste estudo podemos verificar a diminuição de frequência de comportamentos restritos e de estereotipia no grupo experimental, indicando que a cinoterapia foi eficaz na promoção de comportamentos de autorregulação adaptativos. Estes dados são semelhantes a um estudo experimental realizado com crianças internadas revela que as crianças em tratamento com a presença do cão de companhia revelam menos comportamentos ansiógenos do que outras crianças que não têm na sua presença o estímulo canino (Hansen, Messinger, Baun, \& Megel, 1999; Kaminski, Pellino, \& Wish, 2002).

Assim como num estudo de Eddy et al (1988), podemos retirar como resultado deste estudo no grupo experimental que com a presença do cão e a realização da cinoterapia promoveu a diminuição da atividade motora no grupo experimental (e.g. estereotipias) e o aumento da frequência de comportamentos sociais. Assim como neste estudo, também num estudo realizado com crianças se verifica uma associação positiva entre a intervenção com 0 cão de intervenções assistidas por animais e uma melhor aquisição de aprendizagem (Limond, Bradshaw, \& Cormack, 1997). Neste estudo foram tidas em consideração algumas limitações. Uma das limitações prende-se com a não representatividade da amostra relativamente à população geral, pelo que os resultados não devem ser generalizados. Também se considera essencial, em estudos futuros, analisar o ambiente familiar e qual 0 impacto deste no progresso de competências comunicativas, sociais e interpessoais da criança. Os estudos realizados comprovam que as terapias assistidas por animais, no geral, são uma intervenção complementar útil na promoção da melhoria de qualidade de vida de indivíduos com incapacidade intelectual. Também como neste estudo, existem trabalhos que comprovam que com recurso à cinoterapia, como uma terapia complementar, a interação social das crianças com PEA aumenta (Eddy et al., 1988; Esteves \& Stokes, 2008; Muñoz Lasa et al., 2015). Podemos então concluir que a cinoterapia é uma terapia complementar eficaz na promoção de comportamentos de autorregulação, comunicação e socialização de crianças com PEA. Contudo, para testar o custo-benefício destas terapias, são necessários mais estudos com metodologias científico-experimentais (Maber-Aleksandrowicz, Avent, \& Hassiotis, 2016; Muñoz Lasa et al., 2015).

\section{REFERÊNCIAS BIBLIOGRÁFICAS}

APA. (2015). Manual de diagnóstico e estatística das perturbações mentais. (J. C.Fernandes, Ed.) (5th ed.). Lisboa: Climepsi Editores.

Assumpção, F., Kuczynski, E., Gabriel, M. R., \& Rocca, C. C. (1999). Escala da avaliação de traços autisticos (ATA): Validade e confiabilidade de uma escala para a detecção de condutas autísticas. Arquivos de Neuropsiquiatria, 57(1), 23-29. http://doi.org/10.1590/S0004-282X1999000100005

Bronfenbrenner, U. (1994). Ecological models of human development. In International Encyclopedia of Education (2nd ed., Vol. 3, pp. 37-43). Oxford: Elsevier. http://doi.org/http://www.psy.cmu.edu/ siegler/35bronfebrenner94.pdf

Capucha, L. (2008). Unidades de ensino estruturado para alunos com perturbações do espectro do autismo: Normas orientadoras. Lisboa. 
Ciari, M. B., Chelini, M. O. M., Lacerda, J. R., Rocha, C. F. P. G., \& Otta, E. (2013). Animal assisted therapy for autistic children: A pilot study of the evolution of dog/child relationship. Journal of Veterinary Behavior: Clinical Applications and Research, 8(4), e37. http://doi.org/10.1016/j.jveb.2013.04.038

Eddy, J., Hart, L. A., \& Boltz, R. P. (1988). The effects of Service Dogs on social acknowledgments of people in wheelchairs. The Journal of Psychology, 122(1), 39- 45. http://doi.org/10.1080/00223980.1988.10542941

Esteves, S. W., \& Stokes, T. (2008). Social Effects of a Dog 's Presence on Children with Disabilities. Anthrozoos, 21(1), 5-15. http://doi.org/10.1080/08927936.2008.11425166

Fine, A. H. (1999). Handbook on Animal - Assisted Therapy: Theoretical foundations and guidelines for practice. North. http://doi.org/10.1016/B978-0-12-381453-1.10027-3

Fine, A. H. (2010). Animals in the lives of children. In C. S. P. University. (Ed.), Handbook on animal-assisted therapy: Theoretical foundations and Guidelines for Practice. (Third, pp. 225-230). California: Elsevier Inc.

Gabriels, R., Agnew, J., Miller, L. J., Gralla, J., Pan, Z., Goldson, E., ... Hooks, E. (2008). Is there a relationship between restricted, repetitive, stereotyped behaviors and interests and abnormal sensory response in children with autism spectrum disorders?Research in Autism Spectrum Disorders, 2(4), 660-670. http://doi.org/10.1016/j.rasd.2008.02.002

Hansen, K., Messinger, C., Baun, M., \& Megel, M. (1999). Companion animals alleviating distress in children. Anthrozoos: A Multidisciplinary Journal of The Interactions of People \& Animals, 12(3), 142-148. http://doi.org/10.2752/089279399787000264

Kaminski, M., Pellino, T., \& Wish, J. (2002). Play and pets: The physical and emotional impact of child-life and pet therapy on hospitalized children. Children's Health Care, 31(4), 321-335. http://doi.org/10.1207/S15326888CHC3104

Kamioka, H., Okada, S., Tsutani, K., Park, H., Okuizumi, H., Handa, S., ... Mutoh, Y. (2014). Effectiveness of animal-assisted therapy: A systematic review of randomized controlled trials. Complementary Therapies in Medicine, 22(2), 371-390. http://doi.org/10.1016/j.ctim.2013.12.016

Kanner, L. (1943). Austistic disturbances of affective contact. Nervous Child, 2, 217- 250.

Lane, H. B., \& Zavada, S. D. W. (2013). When reading gets ruff: Canine-assisted reading programs. Reading Teacher, 67(2), 87-95. http://doi.org/10.1002/TRTR.1204 Lecavalier, L., Leone, S., \& Wiltz, J. (2006). The impact of behaviour problems on caregiver stress in young people with autism spectrum disorders. Journal of Intellectual Disability Research, 50(3), 172-183. http://doi.org/10.1111/j.1365-2788.2005.00732.x

Leyfer, O., Folstein, S., Bacalman, S., Davis, N., Dinh, E., Morgan, J., ... Lainhart, J. (2006). Comorbid psychiatric disorders in children with autism: Interview development and rates of disorders. Journal of Autism and Developmental Disorders, 36(7), 849-861. http://doi.org/10.1007/s10803-006-0123-0

Limond, J. A., Bradshaw, J. W. S., \& Cormack, K. F. M. (1997). Behavior of children with learning disabilities interacting with a therapy dog. Anthrozoos, 10(2-3), 84- 89. http://doi.org/10.2752/089279397787001139

Maber-Aleksandrowicz, S., Avent, C., \& Hassiotis, A. (2016). A Systematic Review of Animal-Assisted Therapy on Psychosocial Outcomes in People with Intellectual Disability. Research in Developmental Disabilities, 4950, 322-338. http://doi.org/10.1016/j.ridd.2015.12.005

Marinho, S., Gomes, A., Vieira, D., Antunes, E., \& Teixeira, D. (2009). Perturbações do Espectro do Autismo: Avaliação das Competências Comunicativas, Sociais e Linguísticas., 268-281.

Marston, L. (2011). How animals affect us: Examining the influence of human-animal interaction on child development and human health. Anthrozoos: A Multidisciplinary Journal of The Interactions of People \& Animals, 24(3), 339-341. http://doi.org/10.2752/175303711X13080435184438

Matson, J., Gonzalez, M., \& Rivet, T. (2008). Reliability of the Autism Spectrum Disorder-Behavior Problems for Children (ASD-BPC). Research in Autism Spectrum Disorders, 2(4), 696-706. http://doi.org/10.1016/j.rasd.2008.02.003 
Matson, J., Wilkins, J., \& Macken, J. (2008). The Relationship of Challenging Behaviors to Severity and Symptoms of Autism Spectrum Disorders. Journal of Mental Health Research in Intellectual Disabilities, 2(1), 29-44. http://doi.org/10.1080/19315860802611415

Maujean, A., Pepping, C. A., \& Kendall, E. (2015). A systematic review of randomized controlled trials of AnimalAssisted Therapy on psychosocial outcomes. Anthrozoos, 28(1), 23-36. http://doi.org/10.2752/089279315X14129350721812

Muñoz Lasa, S., Máximo Bocanegra, N., Valero Alcaide, R., Atín Arratibel, M. A., Varela Donoso, E., \& Ferriero, G. (2015). Intervenciones asistidas por animales en neurorrehabilitación: Una revisión de la literatura más reciente. Neurologia, 30(1), 1-7. http://doi.org/10.1016/j.nrl.2013.01.012

Pawlik-Popielarska, B. M. (2010). The impact of kynotherapy in handicapped children. Acta Neuropsychologica, 8(1), 29-37.

Redefer, L., \& Goodman, J. (1989). Brief report: Pet-facilitated therapy with autistic children. Journal of Autism and Developmental Disorders, 19(3), 461-467. http://doi.org/10.1007/BF02212943

Reis, H., Pereira, A. P., \& Almeida, L. (2012). Avaliação do perfil desenvolvimental das crianças com perturbação do espectro do autismo: Construção e validação de um instrumento. In II Seminário Internacional: Contributos da Psicologia em Contextos Educativos (pp. 204-212). Braga: Universidade do Minho.

Schieve, L., Blumberg, S., Rice, C., Visser, S., \& Boyle, C. (2007). The Relationship Between Autism and Parenting Stress. Pediatrics, 119(Supplement 1), S114-S121. http://doi.org/10.1542/peds.2006-2089Q

Sousa Magalhães, M. F. (2014). 0 recurso a animais nas intervenções em crianças com Perturbações do Espetro do Autismo. Universidade do Porto.

Thayer, R. E., Branden, N., Fairburn, C., Pitkeathley, J., Emerson, D., Higbee, K. L., ... Kummerow, J. (1998). The autistic spectrum: A guidefor parents and professionals. (1a ed.). Barcelona: Ediciones Paidós Ibérica, S.A.

Viau, R., Arsenault-Lapierre, G., Fecteau, S., Champagne, N., Walker, C. D., \& Lupien, S. (2010). Effect of service dogs on salivary cortisol secretion in autistic children.Psychoneuroendocrinology, 35(8), 1187-1193. http://doi.org/10.1016/j.psyneuen.2010.02.004

White, S., Oswald, D., Ollendick, T., \& Scahill, L. (2009). Anxiety in children and adolescents with autism spectrum disorders. Clinical Psychology Review, 29(3), 216-229. http://doi.org/10.1016/j.cpr.2009.01.003

Yenkoyan, K., Grigoryan, A., Fereshetyan, K., \& Yepremyan, D. (2017). Advances in understanding the pathophysiology of Autism Spectrum Disorders. Behavioural Brain Research, 2-33. http://doi.org/10.1016/j.bbr.2017.04.038 
